\title{
A Survey on Various Enhancements Made on ZRP
}

\author{
Kanishka Raheja \\ Mtech Scholar \\ Department of CSE, \\ BRCM-CET (Bahal), India
}

\author{
Sunil Kr Maakar \\ Assistant professor \\ Department of CSE, \\ BRCM-CET (Bahal), India
}

\begin{abstract}
MANET is a self organizing and self configurable infrastructure less network of mobile nodes connected by wireless where the nodes move arbitrarily. Routing is a critical issue in Mobile Ad Hoc Networks. One of wellknown protocol for Ad hoc networks is Zone Routing Protocol. However, many useless control packets are used resulting in the increase of network load and decrease of network performance. This paper studies various enhancements that have been made on ZRP to improve its performance.
\end{abstract}

\section{Keywords}

MANET, ZRP, cache, IARP, IERP

\section{INTRODUCTION}

A Mobile Ad hoc Network (MANET) is a formation of the network instantaneously and dynamically with the collection of wireless mobile nodes that operates without the support of any fixed network infrastructure [3]. Much work has been done on routing in MANETs .Many protocols and algorithms such as Destination- Sequenced Distance-Vector (DSDV) protocol, Cluster-based routing algorithms, Dynamic Source Routing (DSR) protocol, Ad hoc On-demand Distance-Vector (AODV) protocol, Zone Routing Protocol (ZRP), Temporally Ordered Routing Algorithm (TORA), and Associative Bit Routing (ABR) have been proposed. Among these protocols, the ZRP has a wide application [4]. However, when the protocol searches for a new route, it sends many useless control packets, which increase the network load and decrease the network performance. In this paper we propose various enhancements that are made on ZRP. Existing Ad-hoc routing protocols can be classified into two groups: proactive and reactive routing protocols. Proactive routing protocols attempt to continuously evaluate the routes within the network, so that when a packet needs to be forwarded, the route is already known and can be immediately used. Reactive protocols invoke a route determination procedure on an on-demand basis.

\subsection{ZRP}

The comparison of proactive and reactive scheme shows that there is needed a protocol that combines the advantages of proactive and reactive protocol. The ZRP [1] is a hybrid reactive/proactive scheme. In ZRP, a node proactively maintains routes destinations within a local neighborhood, which is considered as a routing zone. A node routing zone is defined as a collection of nodes whose minimum distance hop from the node is no greater than a parameter referred to as the zone radius. Each node maintains its own routing zone, but the routing zones of neighborhood nodes overlap. If a node want to communicate with in its zone it can communicate proactively and the IntrAzone Routing Protocol (IARP) is used and IntErzone Routing Protocol (IERP) for routing beyond the routing zone.ZRP uses the concept of border casting a node such as if a node cannot find its destination with in a zone it sends the packets to its peripheral nodes of the routing zone and then that node is responsible for searching the destination to its own zone proactively. This process continues until the source finds its destination. The reactive routing process is divided into two phases: the route request phase and the route reply phase. ZRP performs better than any single proactive or reactive protocol [4].But its performance can be further improved by enhancing this protocol. This paper presents various enhancements which have been made in ZRP.

\subsubsection{Analysis}

ZRP is better suitable for the large networks. The performance of protocol has been adjusted accordingly. However, many useless control packets are used resulting in the increase of network load and decrease of network performance. In the reactive protocol, the IERP request packets are sent to all border nodes. The IERP packets are necessary for searching a route. However, these packets may cause a network congestion resulting in the decrease of network performance. In the real communication situations, the data transmission may happen many times to the same DN. But in such situation, in ZRP the border-cast route searching is carried out to all destinations.

\section{A CACHING SCHEME FOR ROUTING IN MOBILE AD HOC NETWORKS AND ITS APPLICATION TO ZRP}

ZRP is based on the notion of a zone. Each node $n$ is the center of a zone with radius $k$ hops, denoted $Z_{k}(n)$. Nodes at a distance lesser than or equal to $k$ from $n$ belong to $Z_{k}(n)$. A proactive protocol is used among the nodes of $Z_{k}(n)$. A reactive protocol is used by $n$ to reach a node outside its zone. Practically, $\mathrm{k}$ is set to a value much smaller than the network diameter to get a fast convergence of the proactive component of ZRP and a low routing protocol overhead. Therefore, the probability that a sender selects a destination out of its zone is high, thus, again, caching is useful to reduce path discoveries of the reactive part of ZRP. Whenever a route to a destination out of the zone is to be discovered then that route is to be cached for further usage [5].

The simplest form of caching is based on timeouts associated with cache entries. When an entry is cached, a timer starts. When the timeout elapses, the entry is removed from the cache. Each time the entry is used, the timer restarts. Therefore, the effectiveness of such a scheme depends on the timeout value associated with a cached route. If the timeout is well-tuned, the protocol performance increases; otherwise, a severe degradation arises as entries are removed either prematurely or too late from the cache. This cache scheme can not only be applied for ZRP but it can be applied for other reactive protocols like AODV. 
After applying this cache scheme with ZRP known as C-ZRP [5], the simulation compared ZRP with C-ZRP. The overall protocol efficiency and the application level performance increases.

\subsection{Analysis}

- Protocol Overhead: The total number of protocol overhead packets generated for each delivered data packet decreases with the extreme case of one discovery per destination as pause time, PT increases.

- Delivery fraction: The ratio of the data packets delivered to the destinations to those generated by the sources. The delivery fraction increases with the pause time, with a reduction in ZRP when using the higher zone's radius.

- Average transmission delay: The elapsed time from when a source has a packet to send until the packet is delivered to the destination shows the average transmission delay as a function of the mobility and different values for the mobility. At low mobility (PT 70\%), delay decreases with the load since the number of route requests decreases. At higher mobility, the delay first decreases for the same reason, but it start to increase when the load reaches a given value, due to an increasing number of path discoveries.

\section{A SELECTIVE BORDER CASTING ZONE ROUTING PROTOCOL FOR AD- HOC NETWORKS}

As already discussed the performance of ZRP is better than other protocols because it combines the advantages of Proactive as well as the reactive scheme. However, many useless control packets are used resulting in the increase of network load and decrease of network performance. A Selective Border -casting Zone Routing Protocol (SBZRP) [1] to reduce the network load by limiting the number of control packets when the protocol searches for a new route. The performance evaluation via simulations shows that the SBZRP has a good behaviour and better performance than ZRP. The proposed SBZRP uses for intra-zone routing the IARP the same as ZRP, but uses a new IERP for inter-zone routing.

The number of the border-cast nodes and IERP packets can be decreased resulting in the increase of the throughput and the decrease of packet mean delay.

\subsection{Analysis}

Performance of SBZRP can be measured on two characteristics:

- Throughput: Throughput or network throughput is the rate of successful message delivery over a communication channel. When the network load is high, the SBZRP has better behaviour than ZRP. When the network load is low, the throughput is decreased for both protocols.

- Packet mean time delay: The delay of a network specifies how long it takes for a bit of data to travel across the network from one node or endpoint to another. When the network load is high, the mean delay of ZRP is higher than SBZRP. we conclude that when the network load and node moving degree is high, the performance of SBZRP is better than ZRP

\section{EXTENDED ZRP: A ROUTING LAYER BASED SERVICE DISCOVERY}

\section{PROTOCOL FOR MOBILE AD-HOC NETWORKS}

The next enhancement that was made on ZRP was the Extended ZRP (E-ZRP), [7] a Routing Layer Based Service Protocol for Mobile Ad Hoc Networks. The enhancement was to add the concept of service discovery which is very essential process in order for these networks to be self configurable with zero or minimal administration overhead. In this adaptation, Service Discovery can be greatly enhanced in terms of efficiency (regarding service discoverability and energy consumption), by piggybacking service information into routing layer messages.

Service discovery protocols (SDP) are network protocols which allow automatic detection of devices and services offered by these devices on a computer networks. The great majority of service discovery protocols developed for MANETs deal with the issues at the application layer. It was argued that by implementing service discovery at the routing layer, instead of the application layer, the resulting communication and energy consumption overheads are significantly reduced. [7][6].

\subsection{E-ZRP}

In order to add service discovery capabilities to ZRP we embedded an extra field in NDP (The Neighbor Discovery Protocol, through which every node periodically broadcasts a "hello" message to denote its presence, "hello" messages for storing service IDs. The concept of Unique Universal Identifiers (UUIDs) was used instead of service descriptions, keeping packet lengths small for the routing messages and minimizing the effects on the network (the bigger the messages the larger the delays and the possibility of transmission errors). ZRP was further extended in order to include service information in every routing entry of the IARP routing messages and tables. IARP listens to information gathered from NDP messages, updates its table and then periodically broadcasts its table to its neighbors.[7]

\subsection{Analysis}

Simulations were conducted through Qualnet Simulator. Two characteristics were measured:

- $\quad$ Service discoverabilities: Average number of discovered services per node proactively is almost the same in both the protocols

- Energy Consumption: Average amount of energy consumed per node in E-ZRP is quite lesser than ZRP.

\section{AN ENHANCED ZONE-BASED ROUTING PROTOCOL FOR MOBILE AD-HOC NETWORKS BASED ON ROUTE RELIABILITY}

To overcome the drawback of ZRP Enhanced Zone -Based Routing Protocol was proposed. In EZRP [8], each node calculates the reliability of the route. In the case of reliable route, the source node sends the data packet directly to the destination node using that route without route searching. While, in the case of unreliable route, the source node searches for a new route again. To adapt this, a Reliable degree parameter was proposed to calculate the root quality in the proposed EZRP. When the RD of the route is high, a data packet is sent directly without route searching. When the RD is middle, a route searching is performed for some limited directions. In this case, we assume that node's movement distance from previous position is small. When the RD is low, 
a route searching is done for all directions the same as the ZRP. For calculating the RD, each node saves searching records ten times for each route [8] [6].

\subsection{Analysis}

Two characteristics were considered while evaluating the performance of EZRP

- Packet arrival rate: The results show that the number of arrived packet to the destination node without loss is higher in EZRP compared to ZRP, resulting in better throughput.

- Average delays: Also the average delay of EZRP is lower than ZRP. Thus it is well suited for highly reliable transmission with higher throughput.

\section{GENETIC ZONE ROUTING PROTOCOL (GZRP)}

The protocol GZRP [9] was the enhancement of ZRP. This protocol applies Genetic Algorithms (GA) to Zone Routing Protocol (ZRP) for finding a limited set of multiple alternative routes to the destinations, in order to provide load balancing and fault tolerance during node/link failure by using the routing database available with the border nodes.

A. Load balancing: Any on-demand routing protocol uses Route Discovery and Route maintenance procedures for finding the path to the destination which in turn return a single shortest path to the destination. In any case, if that route fails and/or congestion occurs and that route leads to the delays or packet losses, then rediscovery of the new SP (Shortest path) is required. This causes unnecessary wastage of network resources and also wastage of time. ZRP is hybrid protocol which has the hands on routes if the destination is within the routing zone. However, the actual problem comes when the destination is outside the zone. In this case, it makes use of Route Discovery with IERP and BRP. This route discovery goes with border nodes between the zones. In our work, we have applied GA at the border nodes in order to find the alternative routes. Instead of rediscovering the path to the destination every time on failure of the existing path due to link/node failure and/or congestion occurrence, the border node will apply GA by making use of topological database available with that node to find alternative routes that may be shortest or near shortest paths[9].

B. Fault tolerance: Fault tolerance is essential in actual routing algorithms. The routing algorithms must be robust for packet loss caused by instability of the network such as congestion and node/link failure. The GZRP reduces this problem by providing a set of alternative routes to a border node. The next best available alternative route at the border node is used for forwarding the packets. This gives robustness for the network and also reduces the control overhead that may occur in the network due to rediscovery of the routes. Also, because there are multiple paths available, the node makes use of these routes in order to load balance the network.[9]

\subsection{Analysis}

- PDR (packet delivery ratio): The GZRP better delivers the packets to the destination as the network size increases, particularly at the higher mobility conditions.
- There is no pause time (high mobility) and no mobility condition, GZRP has shown a reduction in delay value for 100 nodes network.

- There is an improvement in the reduction of control overhead in the 100-node network due to GZRP up to $10 \%$.

\section{A SWARM-BASED HYBRID ROUTING PROTOCOL TO SUPPORT MULTIPLE QUALITY OF SERVICE (QOS) METRICS IN MOBILE AD HOC NETWORKS}

ZRP was enhanced to provide Quality of service which is defined as a set of service requirements that require to be met by the network while transporting the data packets from source to destination. The network is expected to guarantee a set of quantitatively measurable attributes to the user in terms of end-to-end delay, bandwidth, probability of packet loss, energy and delay variance (jitter). ZRP with Ant Colony Optimization (ACO) [11] is adapted which is a heuristic approach for solving hard combinatorial optimization problems. The basic idea behind ACO algorithms for routing is the use of mobile agents, called ants. These ants are generated by nodes in the network, with the task to sample a path between the node and an assigned destination. An ant going from source node to destination node collects information about the quality of the path (e.g. round trip time, number of hops, hop delay, available bandwidth, node energy etc.) using pheromone variables, and uses this on its way back from destination to update the routing information at the intermediate nodes. The idea in Ant Net is to use two different network exploration agents (forward and backward ants), which collect information about delay, congestion status and the followed path in the network. Ant based Control (ABC) is another proactive ant based algorithm designed for telephone networks. Ant Colony Based Routing Algorithm (ARA) works in an on-demand way, with ants setting up multiple paths between source and destination at the start of a data session. Probabilistic Emergent Routing Algorithm (PERA) works in an on-demand way, with ants being broadcast towards the destination at the start of a data session. Multiple paths are set up, but only the one with the highest pheromone value is used by data and the other paths are available for backup. Two pheromone tables AntIntrazone Pheromone Table (AntIntraPT) and AntInterzone pheromone Table (AntInterPT) are implemented for each node. The AntIntraPT is proactively maintained so that a node can obtain a path to any node within its zone quickly. The AntInterPT stores the path to a node beyond its zone and is setup on demand as routes outside a zone is required. The peripheral nodes of the zone are used to find routes between zones. [10]

\subsection{Analysis}

Three characteristics were measured through the simulation:

- $\quad$ Packet Delivery ratio (PDR): Shows how successfully packets are delivered to its destination and in that results showed that SHRP performs better than ZRP.

- Average end to end delay: From 50 nodes to 100 nodes, there is an increase in delay which may be due to the insufficient number of nodes for proper zone formation. After 100 nodes, the delay is gradually reducing.

- $\quad$ Routing protocol overhead: SHRP is having slightly high overhead than that of ZRP which is due to the additional 
control packets or increase in the size of the control packet.

\section{CONCLUSION}

This paper reviews various enhancements that are made on ZRP. The paper also discusses analysis of these protocols. Different protocols have different advantages and disadvantages under various circumstances. The study contains various schemes like caching, Extended ZRP, genetic, swarm based techniques etc. The study shows that the improvement in PDR or Average end to end delay enhances the routing overhead of the protocol by applying ACO on ZRP. In future ZRP can be enhanced to improve PDR or by applying security by removing antnet from Interzone, while maintaining the routing overhead.

\section{REFERENCES}

[1] Leonard Barolli, Yoshitaka Honma, Akio Koyama, Arjan Durresi and Junpei Arai. 2004. A Selective Bordercasting Zone Routing Protocol for Ad-hoc Networks. Proceedings of the 15th International Workshop on Database and Expert Systems Applications (DEXA'04)

[2] Hoebeke, J., Moerman, I., Dhoedt, B., \& Demeester, P. 2004. An overview of mobile ad hoc networks: Applications and challenges. Journal-Communications Network, 3(3), 60-66.

[3] P. Sateesh Kumar,Dr.S. Ramachandram. 2008. Scalability of Network Size on Genetic Zone Routing Protocol for MANETs. International Conference on Advanced Computer Theory and Engineering.

[4] Beijar, N. 2002. Zone routing protocol (ZRP). Networking Laboratory, Helsinki University of Technology, Finland.

[5] Roberto Beraldi and Roberto Baldoni.2003.A Caching Scheme for Routing in Mobile Ad Hoc Networks and Its Application to ZRP. IEEE TRANSACTIONS ON COMPUTERS, VOL. 52, NO. 8,2003.

[6] Dhanya Sudarsan, Jisha G.2012. A Survey on Various Improvements of Hybrid Zone Routing Protocol in MANET. International Conference on Advances in Computing, Communications and Informatics (ICACCI2012).page 1261-1265.

[7] Christopher N. Ververidis and George C. Polyzos.2005. Extended ZRP: a Routing Layer Based Service
Discovery Protocol for Mobile Ad Hoc Networks. Proceedings of the Second Annual International Conference on Mobile and Ubiquitous Systems: Networking and Services (MobiQuitous'05).

[8] Akio Koyama, Yoshitaka Honma, Junpei Arai, Leonard Barolli.2006.An Enhanced Zone-Based Routing Protocol for Mobile Ad-Hoc Networks Based on Route Reliability. Proceedings of the 20th International Conference on Advanced Information Networking and Applications (AINA'06).

[9] P. Sateesh Kumar, Dr.S. Ramachandram. 2008 Scalability of Network Size on Genetic Zone Routing Protocol for MANETs. International Conference on Advanced Computer Theory and Engineering.

[10] S.K.Nivetha，R.Asokan andN.Senthilkumaran.2013. A Swarm-based Hybrid Routing Protocol to Support Multiple Quality of Service (QoS) Metrics in Mobile Ad Hoc Networks. 4th ICCCNT 2013. IEEE - 31661

[11] Prince Samar, Marc R. Pearlman, and Zygmunt J. Haas.2004. Independent Zone Routing: An Adaptive Hybrid Routing Framework for Ad Hoc Wireless Networks. IEEE/ACM Transactions on Networking, Vol. 12, No. 4, 2004.

[12] G.Di Caro, and M. Dorigo.1988.AntNet: Distributed stigmergetic control for communications networks. Journal of Artificial Intelligence Research, 9:317-365, 1988.

[13] Dilli Ravilla, Dr Chandra Shekar Reddy Putta.2013.Performance of Secured Zone Routing Protocol due to the Effect of Malicious Nodes in MANETs. 4th ICCCNT 2013. IEEE - 31661.

[14] Sandeep Kaur, Supreet Kaur. 2013. ANALYSIS OF ZONE ROUTING PROTOCOL IN MANET. IJRET: International Journal of Research in Engineering and Technology. Volume: 02 Issue: 09.

[15] SreeRangaRaju,M.N.Mungara,Jitendranath.2010. Performance evaluation of ZRP in adhoc mobile wireless network using Qualnet simulator. Signal Processing and Information Technology (ISSPIT), IEEE International Symposium on 2010 , Page(s): 457 - 466

[16] http://en.wikipedia.org/wiki/Zone_Routing_Protocol 